\title{
Pre-trial Gaze Stability Predicts Momentary Slips of Attention
}

\author{
Pre-trial Gaze Stability and Task focus
}

Han Zhang*

Department of Psychology, University of Michigan, hanzh@umich.edu

John Jonides

Department of Psychology, University of Michigan, jjonides@umich.edu

Our ability to maintain focus on a task waxes and wanes. Recent research suggests that eye-tracking may be a useful tool to capture the momentary slips of attention. In this paper, we show that pre-trial gaze stability predicted momentary slips of attention on the upcoming trial. In two visual search tasks, we asked participants to stabilize their gaze on a fixation cross before the search array appeared. We recorded participants' manual responses and eye movements as they searched for the target. We also occasionally presented thought probes to examine whether participants were mind-wandering on a subset of trials. Results from the two tasks revealed a converging pattern: lower pre-trial gaze stability predicted worse performance and greater self-reported mind-wandering in the upcoming trial. These findings suggest that pre-trial gaze stability is a simple and objective measure that may capture moments of inattention. It may be used to curb the negative effects of inattention before the actual task starts.

CCS CONCEPTS • Human-centered computing • Human computer interaction (HCI) • HCI design and evaluation methods

Additional Keywords and Phrases: Eye-tracking, attention, mind-wandering, visual search

\section{ACM Reference Format:}

Han Zhang and John Jonides. 2021. Pre-trial Gaze Stability Predicts Momentary Slips of Attention. In EMICS '21: ACM CHI '21 Workshop on Eye Movements as an Interface to Cognitive State, May 14, 2021, Yokohama, Japan. ACM, New York, NY, USA, x pages.

\section{INTRODUCTION}

It is notoriously difficult to sustain attention for a long period of time. In a typical cognitive task, participants are repeatedly exposed to a similar set of trials, and each time they must respond based on a certain task goal (e.g., find the target). Situations like this are conducive to mind-wandering, an attentional state in which one engages in thoughts unrelated to the current task [1]. Studies such as [2], [3] have reported that mind-wandering is ubiquitous in both laboratory and real-life settings and is often associated with compromised task performance.

* Permission to make digital or hard copies of part or all of this work for personal or classroom use is granted without fee provided that copies are not made or distributed for profit or commercial advantage and that copies bear this notice and the full citation on the first page. Copyrights for third-party components of this work must be honored. For all other uses, contact the owner/author(s).

EMICS '21, May 14, 2021, Yokohama, Japan

(C) 2021 Copyright held by the owner/author(s). 
Mind-wandering is traditionally measured via thought-sampling techniques. That is, participants are occasionally presented with a thought probe asking about their current thoughts and focus [4]. The method has been used extensively in the literature, but some noteworthy drawbacks including the reliance on self-reports and being too obtrusive to the primary task [5], [6]. These drawbacks have prompted calls for new measures of mindwandering beyond self-reports [7].

Recently, eye-tracking has begun to gain traction as an alternative measure of one's attentional state. Compared to thought-sampling, eye-tracking is objective, unobtrusive, and provides a continuous measurement. Studies have found that episodes of self-reported mind-wandering are correlated with changes in certain eye-tracking parameters, such as reduced fixation count and increased fixation durations [8], [9]. There is also some limited but encouraging success in using machine learning algorithms to predict mind-wandering based on eye-tracking measures [10], [11]. However, the findings from this work are not entirely consistent across studies [12], [13], [14]. Moreover, the measured eye movement patterns were obtained while participants completed the actual task (i.e., viewing a picture or reading a sentence). If mind-wandering can be detected before the start of the task, we may have the opportunity to proactively mitigate its negative consequences to the primary task.

In this paper, we show that pre-trial gaze stability could signal mind-wandering in the upcoming trial. When participants were asked to fixate on a cross before the start of a trial, having unstable gaze positions predicted worse performance and greater mind-wandering in the upcoming trial. Previous research has shown that mindwandering is associated with motor restlessness (e.g., fidgeting) and poor inhibition of behavior [15], [16]. Here we sought to extend those findings to oculomotor control. The idea that gaze stability could signal mind-wandering also receives theoretical support. In particular, the attentional resources account proposes that during mindwandering, few attentional resources will be directed to support the primary task [17]. To the extent that stabilizing the gaze also requires attentional resources, participants would be less able to do it during mind-wandering.

\section{METHODS}

\subsection{Participants}

Thirty-two undergraduate students from the authors' institution participated in Study 1. Data from 7 participants were excluded $^{1}$. The final sample consisted of 25 participants (Mean Age $=19.12$, SD Age $=1.11,48 \%$ female). An independent sample of 49 undergraduate students participated in Study 2. Data from 9 participants were excluded. The final sample consisted of 40 participants (Mean Age $=18.95$, SD Age $=1.04,60 \%$ female).

\subsection{Apparatus and Tasks}

Participants completed each task in a quiet room with dimmed lighting. The tasks were presented on a desktop computer with a 20.1-inch screen at a distance of approximately 80 centimeters from the participants. Monocular eye movements were recorded by an EyeLink 1000 System at a $500 \mathrm{~Hz}$ sampling rate. Head movements were controlled by tracking a sticker placed on the participants' forehead in Study 1 and by using a chin rest in Study 2 .

Study 1's task was adapted from the additional singleton paradigm [18]. During the pre-trial interval, participants had to maintain their gaze within a $2^{\circ}$ radius of a fixation cross $\left(.86^{\circ *} .86^{\circ}\right)$ presented at the center of the screen for a total of $1000 \mathrm{~ms}$ to trigger the search array. The search array consisted of 10 unfilled shapes

1 Study 1: (1) pre-existing medical conditions $(n=1),(2)$ incomplete data $(n=3),(3)$ technical errors during the experiment $(n=1),(4)$ or not following instructions $(n=2)$. Study 2: (1) pre-existing medical conditions $(n=3)$, and (2) incomplete data $(n=6)$. 
distributed equally on an imaginary circle with a radius of $9.15^{\circ}$. Items consisted of unfilled diamonds $\left(1.71^{\circ}\right.$ diagonal) and circles ( $1.50^{\circ}$ diameter), with the outline drawn in either red or green. Inside each item, there was a line segment $\left(.63^{\circ *} .11^{\circ}\right)$ drawn in grey. The task was to identify the oddly shaped item and indicate whether the line inside the item was vertical or horizontal. In half of the trials, one randomly selected non-target was drawn in a different color from the rest. Participants were told to ignore the distractor and respond as quickly and as accurately as possible. Participants first completed a practice block of 20 trials before 5 experimental blocks of 160 trials each. The eye-tracker was calibrated at the beginning of each block. Study 2's task was a variation of the additional singleton paradigm in which participants searched for a specific shape among heterogeneous items [19]. All aspects of the task were identical to Study 1 except for the following: Participants had to maintain their gaze within a $1.5^{\circ}$ radius of a fixation cross $\left(.63^{\circ *} .57^{\circ}\right)$ for a total of $2000 \mathrm{~ms}$ to trigger the search array. The search array consisted of 6 filled shapes distributed equally on an imaginary circle with a radius of $4.5^{\circ}$. The target was always a diamond $\left(1.13^{\circ}\right.$ diagonal) or a circle $\left(0.9^{\circ}\right.$ diameter $)$, counterbalanced across participants. Non-target items consisted of squares, hexagons, and the unselected target item (i.e., circle for the diamond group, diamond for the circle group). The line segment $\left(.19^{\circ *} .05^{\circ}\right)$ inside each item was drawn in black. Participants completed a practice block of 80 trials and then 6 experimental blocks of 80 trials each.

\subsection{Thought Probes}

In Study 1, thought probes occurred after a haphazard $10 \%$ of the trials asking whether during the previous trial participants were on-task (focusing on completing the task and not thinking about things unrelated to the task) or off-task (thinking about something completely unrelated to the task). In Study 2, thought probes also occurred after $10 \%$ of the trials asking participants whether during the previous trial they were on-task, unintentionally mindwandering (thinking about something completely unrelated to the task despite your best intention to stay on task), or intentionally mind-wandering (decided to think about things completely unrelated to the task) [20].

\subsection{Data Preprocessing and Analysis}

Fixations and saccades were detected using Eyelink's proprietary algorithm. Fixations and saccades with landing positions outside of the screen center ( $>4.5^{\circ}$ of visual angle in S1 and $>2.5^{\circ}$ of visual angle in S2) were assigned to be on the target, the distractor, and other items based on the nearest item. Pre-trial gaze stability was defined as the root mean square of the Euclidean distance from each gaze sample's position to the average position of all gaze samples during the pre-trial interval. For each participant, we created deciles that divided each participant's gaze stability distribution into ten equal parts. We examined the following measures: (1) response times (correct trials only), (2) the number of fixations, (3) mean duration of fixations, (4) probability of capture (\% of trials with the first saccade on the distractor; distractor-present trials only), (5) target looking time (the sum of fixations on the target), and (6) mind-wandering rate (the percentage of thought probes with a mind-wandering response, regardless of whether it was intentional or unintentional; probed trials only). Data analysis was performed in $\mathrm{R}$ using linear mixed models [18]. Random intercepts and slopes for each participant were included in all models.

\section{RESULTS}

Results of the two studies revealed a converging pattern (see Figure 1): Lower pre-trial gaze stability predicted worse performance and greater mind-wandering on the upcoming trial. Specifically, participants took longer time to respond, appeared to be more by influenced the distractor, and inspected the target for a longer duration. In 
Figure 1, the mind-wandering rate was the proportion of mind-wandering responses within each gaze stability decile. To further evaluate the prediction performance of gaze stability, we refitted a generalized logistic regression model with mind-wandering as a binary variable. We then set the cut-off value of .5 and obtained a confusion matrix of the model. For Study 1, we obtained a hit rate of $50.63 \%$, a specificity of $82.7 \%$, a precision of $62.87 \%$, and a $d^{\prime}$ of .96. For Study 2, we obtained a hit rate of $52.58 \%$, a specificity of $83.23 \%$, a precision of $66.02 \%$, and a $d^{\prime}$ of 1.03 .
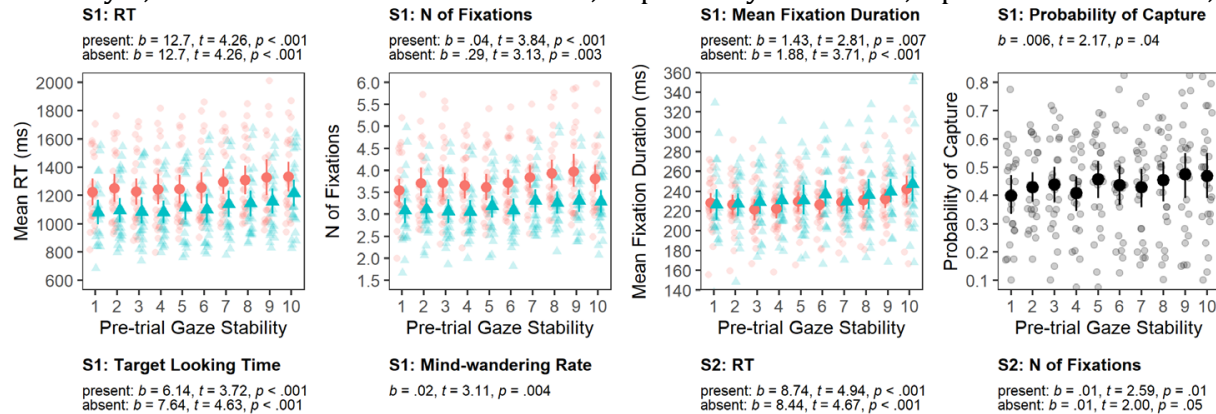

S1: Mind-wandering Rate

$$
\text { S2: } \mathbf{R}
$$
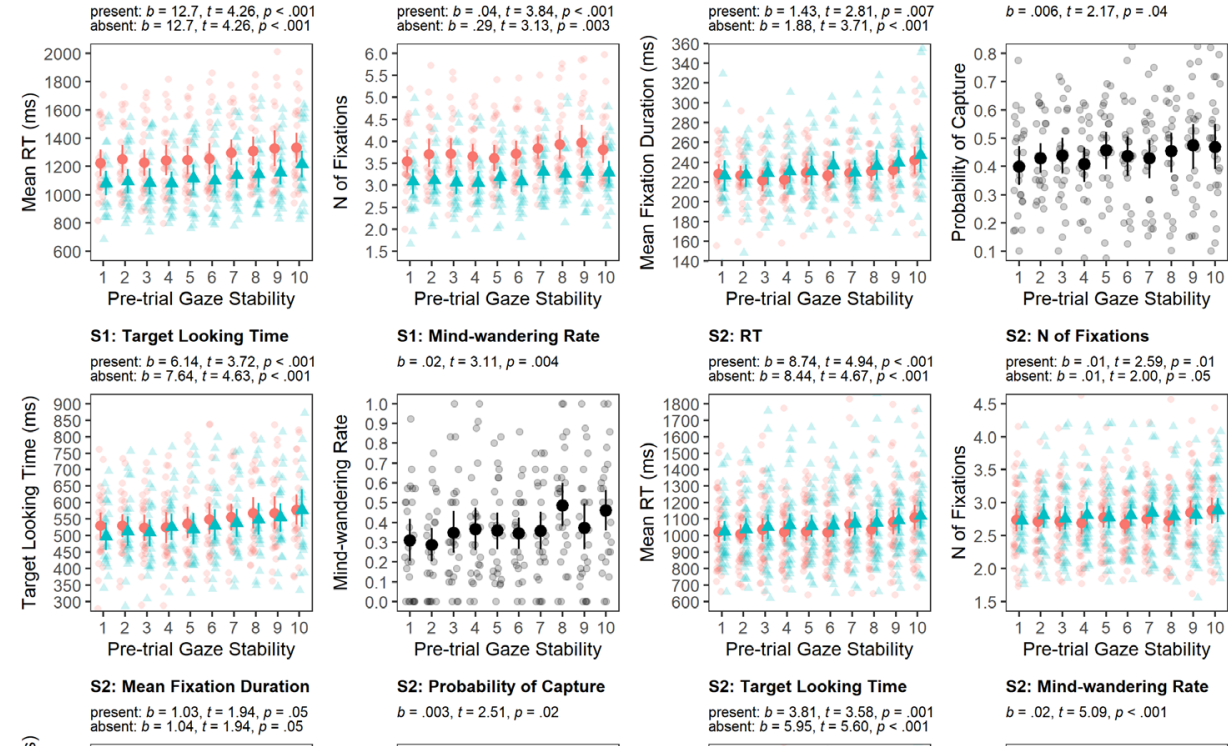

S2: Probability of Capture

$b=.003, t=2.51, p=.02$
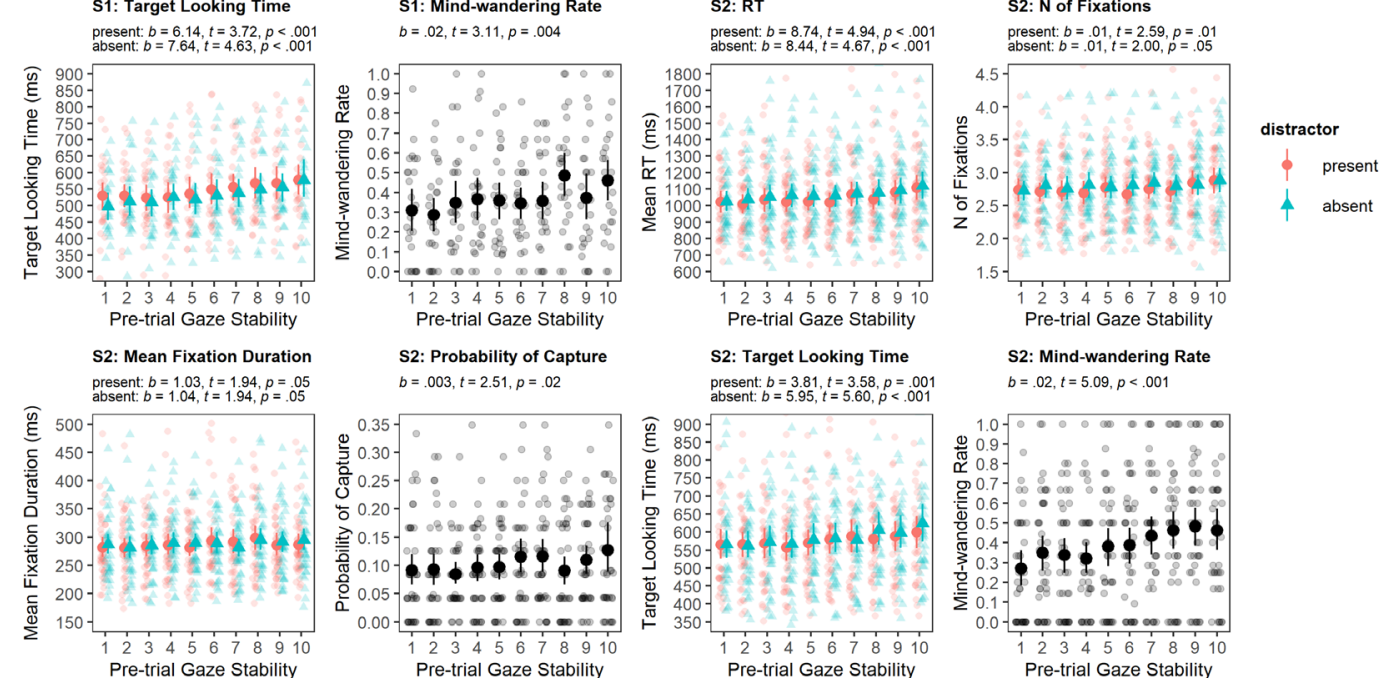

Figure 1: Pre-trial gaze stability deciles (1: the most stable, 10: the most unstable) predicting various measures of Study 1 and 2's visual search tasks. Error bars show 95\% confidence intervals around the mean. Estimated linear trends are shown above each plot.

\section{DISCUSSION}

The results revealed that pre-trial gaze stability, as measured by the dispersion of pre-trial gaze locations, could signal participants' attentional state. Across two visual search tasks, lower pre-trial gaze stability consistently predicted worse performance on the upcoming trial and greater self-reported mind-wandering. The calculation of gaze stability is easy and can be integrated with many cognitive tasks. Also, because the measure is taken before rather than during the actual task, it allows us to proactively address the impact of inattention rather than reacting to it once it has caused damage to the task. Note that there are still miles to run before we can detect inattention in real-time. For one thing, it is unclear whether linear mixed model is the optimal algorithm to deploy in a wearable device for real-time detection. We also do not yet know whether the results are robust to new data. But we do hope that the current study will pique interest for more thorough investigations on this topic in the near future. 


\section{REFERENCES}

[1] J. Smallwood and J. W. Schooler, “The restless mind.," Psychol. Bull., vol. 132, no. 6, pp. 946-958, Nov. 2006, doi: 10.1037/0033-2909.132.6.946.

[2] M. J. Kane et al., "For Whom the Mind Wanders, and When, Varies Across Laboratory and Daily-Life Settings," Psychol. Sci., vol. 28, no. 9, pp. 1271-1289, Sep. 2017, doi: 10.1177/0956797617706086.

[3] B. W. Mooneyham and J. W. Schooler, "The costs and benefits of mind-wandering: A review.," Can. J. Exp. Psychol. Can. Psychol. Expérimentale, vol. 67, no. 1, pp. 11-18, 2013, doi: 10.1037/a0031569.

[4] J. Smallwood and J. Schooler, "The Science of Mind Wandering: Empirically Navigating the Stream of Consciousness," Annu. Rev. Psychol., vol. 66, no. 1, pp. 487-518, 2015, doi: 10.1146/annurev-psych-010814-015331.

[5] Y. Weinstein, "Mind-wandering, how do I measure thee with probes? Let me count the ways," Behav. Res. Methods, vol. 50, no. 2, pp. 642-661, Apr. 2018, doi: 10.3758/s13428-017-0891-9.

[6] P. Seli, J. S. A. Carriere, M. Levene, and D. Smilek, "How few and far between? Examining the effects of probe rate on self-reported mind wandering," Front. Psychol., vol. 4, 2013, doi: 10.3389/fpsyg.2013.00430.

[7] J. Smallwood, "Distinguishing how from why the mind wanders: A process-occurrence framework for self-generated mental activity.," Psychol. Bull., vol. 139, no. 3, pp. 519-535, May 2013, doi: 10.1037/a0030010.

[8] K. Krasich, R. McManus, S. Hutt, M. Faber, S. K. D’Mello, and J. R. Brockmole, “Gaze-Based Signatures of Mind Wandering During Real-World Scene Processing," J. Exp. Psychol. Gen., vol. 147, no. 8, pp. 1111-1124, 2018, doi: 10.1037/xge0000411.

[9] E. D. Reichle, A. E. Reineberg, and J. W. Schooler, “Eye movements during mindless reading," Psychol. Sci., vol. 21, no. 9, pp. 1300-1310, 2010.

[10] R. Bixler and S. D'Mello, “Automatic gaze-based user-independent detection of mind wandering during computerized reading," User Model. User-Adapt. Interact., vol. 26, no. 1, pp. 33-68, 2016, doi: 10.1007/s11257-015-9167-1.

[11] M. Faber, R. Bixler, and S. K. D'Mello, "An automated behavioral measure of mind wandering during computerized reading," Behav. Res. Methods, vol. 50, no. 1, pp. 134-150, Feb. 2018, doi: 10.3758/s13428-017-0857-y.

[12] M. Faber, K. Krasich, R. Bixler, J. Brockmole, and S. D'Mello, “The eye-mind wandering link: identifying gaze indices of mind wandering across tasks," J. Exp. Psychol. Hum. Percept. Perform., 2020.

[13] H. Zhang, N. C. Anderson, and K. F. Miller, "Refixation patterns of mind-wandering during real-world scene perception," J. Exp. Psychol. Hum. Percept. Perform., vol. 47, no. 1, pp. 36-52, 2021, doi: 10.1037/xhp0000877.

[14] L. Steindorf and J. Rummel, "Do your eyes give you away? A validation study of eye-movement measures used as indicators for mindless reading," Behav. Res. Methods, Feb. 2019, doi: 10.3758/s13428-019-01214-4.

[15] Seli, P., Carriere, J. S., Thomson, D. R., Cheyne, J. A., Martens, K. A. E., \& Smilek, D. (2014). Restless mind, restless body. Journal of experimental psychology: learning, memory, and cognition, 40(3), 660.

[16] McVay, J. C., \& Kane, M. J. (2012). Drifting from slow to “d'oh!": Working memory capacity and mind wandering predict extreme reaction times and executive control errors. Journal of Experimental Psychology: Learning, Memory, and Cognition, 38(3), 525.

[17] Smallwood, J., \& Schooler, J. W. (2006). The restless mind. Psychological bulletin, 132(6), 946.

[18] J. Theeuwes, "Perceptual selectivity for color and form," Percept. Psychophys., vol. 51, no. 6, pp. 599-606, Nov. 1992, doi: 10.3758/BF03211656

[19] N. Gaspelin, C. J. Leonard, and S. J. Luck, "Suppression of overt attentional capture by salient-but-irrelevant color singletons," Atten. Percept. Psychophys., vol. 79, no. 1, pp. 45-62, Jan. 2017, doi: 10.3758/s13414-016-1209-1.

[20] P. Seli, E. F. Risko, D. Smilek, and D. L. Schacter, “Mind-Wandering With and Without Intention,” Trends Cogn. Sci., vol. 20, no. 8, pp. 605-617, Aug. 2016, doi: 10.1016/j.tics.2016.05.010. 